\title{
Vielfältige Anwendungen genetischer Methoden im Wald
}

Rolf Holderegger
Eidgenössische Forschungsanstalt für Wald, Schnee und Landschaft $(\mathrm{CH})^{*}$

\begin{abstract}
Vielfältige Anwendungen genetischer Methoden im Wald
Der Schweizer Wald wird naturnah bewirtschaftet und weitgehend natürlich verjüngt. Dies bietet gute Voraussetzungen für die Erhaltung der genetischen Vielfalt von Baumarten. Wozu also Genetik, wenn Baumzüchtung und -pflanzung im Schweizer Wald keine grosse Bedeutung haben? Tatsächlich sind die Anwendungen genetischer Methoden im Wald vielfältig. Eingesetzt werden sie beispielsweise zur Erfassung von Vernetzung und zur Kontrolle des Erfolgs von Vernetzungsmassnahmen, etwa zwischen Naturwaldreservaten, Altholzinseln und Habitatbäumen. Mit genetischen Methoden kann auch untersucht werden, wie sich einheimische Baumarten und deren Genvarianten nutzen lassen, um den Wald an den Klimawandel anzupassen. Weitere Einsatzbereiche sind die Erforschung der Biodiversität und der Ökologie, etwa des Einflusses der Waldwirtschaft auf die Bodenbiodiversität, oder die Bestimmung von nicht einheimischen Schadorganismen und die Suche nach biologischen Bekämpfungsmethoden. Im Rahmen der multifunktionalen Waldbewirtschaftung können genetische Methoden somit eine wichtige Rolle spielen und sinnvoll eingesetzt werden.
\end{abstract}

Keywords: ecological genetics, adaptation to climate change, forest biodiversity, invasive pests and pathogens, habitat connectivity, forest trees

doi: $10.3188 /$ szf.2016.0308

* Zürcherstrasse 111, CH-8903 Birmensdorf, E-Mail rolf.holderegger@wsl.ch

G enetik im Wald, da denkt man an Züchtung von wirtschaftlich wichtigen Baumarten und an entsprechende Pflanzungen (Sehr \& Fluch 2016, dieses Heft). Wie wichtig ist aber Züchtung und Pflanzung im Schweizer Wald? Der Schweizer Wald wird zu 90\% natürlich verjüngt (Rigling \& Schaffner 2015). Pflanzungen nehmen weiterhin $\mathrm{ab}$ und werden vor allem nach Sturmschäden, im Schutzwald oder zur Erhöhung der Biodiversität vorgenommen. Die naturnahe Waldbewirtschaftung führt zu einer immer natürlicheren Baumartenzusammensetzung im Schweizer Wald. Die Bestände seltener Baumarten werden in Sonderwaldreservaten gefördert, und in Naturwaldreservaten dürfen natürliche Prozesse frei ablaufen (Rigling \& Schaffner 2015). Dies alles benötigt keine Züchtung und kaum Pflanzungen und bietet beste Voraussetzungen für die Erhaltung der genetischen Ressourcen einheimischer Baumarten.

Wo sind dann die Anwendungen der Genetik im Schweizer Wald? In diesem breit gefächerten und bewusst einfach gehaltenen Einstiegsartikel wird auf vier verschiedene Anwendungsbereiche eingegan- gen, zu denen die Genetik wesentliche Beiträge liefert: 1) die Vernetzung von Lebensräumen, 2) die Anpassung von Bäumen an den Klimawandel, 3) das Biodiversitätsmonitoring und 4) die Bestimmung und Bekämpfung von invasiven Schadorganismen. Genauere Informationen finden sich in den folgenden Artikeln dieses Hefts bzw. in der angeführten Literatur.

Eines vorweg: Dank grossen technischen Fortschritten haben sich in den letzten Jahren ganz neue Möglichkeiten für genetische Untersuchungen in Forschung und Praxis eröffnet. 2003 wurde das Erbgut des Menschen vollständig entschlüsselt. Dies nach fast zwei Jahrzehnten Arbeit und schätzungsweise drei Milliarden Dollar Gesamtkosten. Heute, nur etwas mehr als zehn Jahre später, wäre die vollständige Entschlüsselung des Erbguts von 100 Bäumen mit Laborkosten von lediglich einigen Zehntausend Franken $\mathrm{zu}$ bewerkstelligen, und dies obschon Bäume meist ein grösseres Erbgut als der Mensch besitzen. Das Stichwort hierfür ist «NextGeneration Sequencing». Darunter wird eine Vielzahl technischer Analysemöglichkeiten zusammen- 

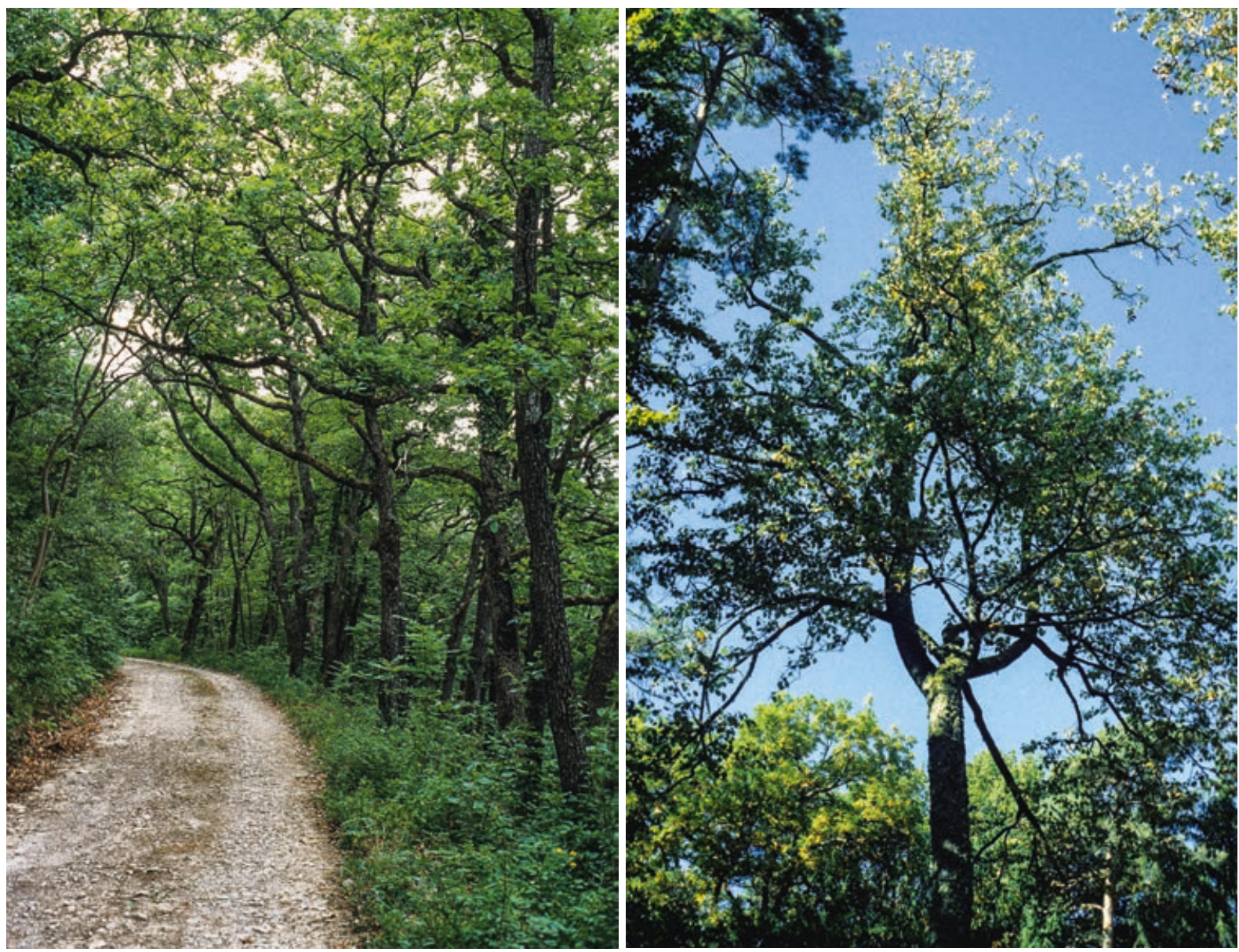

Abb 1 Natur- und Sonderwaldreservate (Flaumeichenwald, Osterfingen SH, links) dienen dem Prozessschutz und dem Schutz der Biodiversität. Speierling (Sorbus domestica, rechts) als Beispiel für eine in Sonderwaldreservaten vorkommende lichtbedürftige, seltene Baumart.

gefasst, die alle in die gleiche Richtung zielen: immer mehr und immer genauere genetische Daten immer billiger herzustellen.

\section{Vernetzung von Lebensräumen}

Grüne oder ökologische Infrastruktur ist eines der Hauptthemen der Biodiversitätsstrategie der Schweiz (BAFU 2012). Ökologische Infrastruktur besteht aus Schutz- oder speziellen Fördergebieten und aus Verbindungselementen. Mithilfe eines Netzes von Lebensräumen und Korridoren soll ein Lebensraumverbund hergestellt werden, welcher den Austausch von Tieren, Pflanzen und Pilzen und somit auch von Genen sichert. Das gilt auch für den Wald. Betrachtet man etwa die grüne Infrastruktur später Waldentwicklungsphasen, so beinhaltet diese die Naturwaldreservate als Eckpfeiler des Netzwerks, seit Langem nicht mehr bewirtschaftete Wälder und Altholzinseln als Trittsteine dazwischen und Habitatbäume als Elemente, welche die allgemeine Durchlässigkeit des Waldes für Arten alter Wälder fördern. Dabei stellen sich Fragen wie: Wie weit entfernt voneinander dürfen Naturwaldreservate liegen, damit sie noch miteinander vernetzt sind? Welche Dichte müssen Altholzinseln oder Habitatbäume aufweisen, damit sie effektiv als Verbindungselemente für den Austausch von Individuen und Genen funktionieren? Ähnliche Fragen lassen sich auch für Sonderwaldreservate oder lichte Wälder stellen (Abbildung 1, links).

Wie kann Vernetzung überprüft werden? Der herkömmliche Ansatz erfasst die Artengemeinschaft an verschiedenen Orten unter jeweils unterschiedlichen Bedingungen. Ein Beispiel wären Naturwaldreservate, welche über Altholzinseln strukturell miteinander verbunden sind oder dies eben nicht sind. Ist die Artengemeinschaft an allen Orten ähnlich, so wird angenommen, dass die Naturwaldreservate auch ohne Altholzinseln miteinander vernetzt sind. Der Prozess selbst, also der Austausch von Individuen, wird dabei gar nicht untersucht. Mehr Information würde man erhalten, wenn die Besiedlung neu geschaffener Lebensräume beobachtet werden könnte, also zum Beispiel neue Vorkommen von holzbewohnenden Insekten oder Pilzen in Naturwaldreservaten. Leider sind Neubesiedlungen seltene Ereignisse; sie lassen sich darum nur schwer erfassen. Grössere Tiere kann man mit Radio- oder GPS-Sendern versehen. Man verfolgt dann, wie sie verschiedene Landschaftselemente nutzen. Da solche Besenderungen teuer sind, lassen sich meist nur wenige Individuen über kurze Zeiträume untersuchen. Bei der Vielzahl 


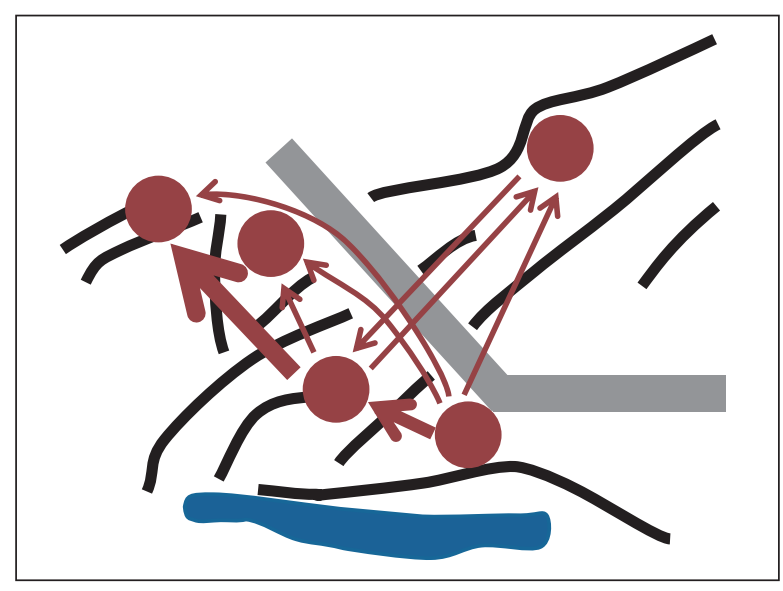

Abb 2 Wanderungen des Auerhuhns (Tetrao urogallus) zwischen Beständen (rote Punkte) im Toggenburg (blau: Walensee; schwarz: Bergketten; grau: Talboden des Toggenburgs) innerhalb einer Generation. Rote Pfeile geben die Anzahl und die Richtung von Individuen an, die zwischen Beständen gewandert sind (dünne Pfeile: ein wanderndes Individuum; mittel dicker Pfeil: zwei; dicker Pfeil: drei wandernde Individuen). Verändert nach Kormann et al (2012).

der kleinen Tiere, bei Baumarten, anderen Pflanzen, Pilzen und Flechten ist eine Besenderung nicht möglich. Wo aber Samen, Pollen und Sporen sich ausbreiten bzw. Tiere wandern, da wandern auch Gene, und dies lässt sich mittels Genetik nachweisen.

Für Baumarten verfügen wir über viele entsprechende genetische Untersuchungen aus der ganzen Welt (Bacles \& Jump 2011) und speziell auch aus der Schweiz. Dies gilt für seltene Baumarten wie Wildbirne (Pyrus pyraster), Elsbeere (Sorbus torminalis) oder Speierling (Sorbus domestica), aber auch für häufige Arten wie Buche (Fagus sylvatica) oder Eichen (Quercus sp.). Beim Speierling (Abbildung 1, rechts) konnte mit einer Vaterschaftsanalyse nachgewiesen werden, dass Pollen (durch Insekten) bis zu $16 \mathrm{~km}$ und Samen (durch Wildtiere) bis zu $12 \mathrm{~km}$ weit verbreitet werden (Kamm et al 2012). Mit anderen Worten: Selbst bei einer so seltenen und zerstreut wachsenden Baumart wie dem Speierling sind die einzelnen Individuen oder Teilvorkommen in einer Landschaft miteinander vernetzt. Betrachtet man lediglich die Vorkommen des Speierlings in der Landschaft, würde man das nie vermuten. Das Problem vieler seltener Baumarten ist damit kaum die Vernetzung, sondern die Fruchtbildung und die Naturverjüngung, die oft fehlen. Ganz ähnliche Ergebnisse hat man an vielen Orten weltweit gefunden (Kramer et al 2008): Baumbestände scheinen in aller Regel über mehrere Kilometer hinweg durch Pollen- und Samenaustausch vernetzt zu sein. Eine erfreuliche Botschaft, die zeigt, dass grosse und kleine Bestände von Baumarten oft in einen Lebensraumverbund eingebunden sind.

Entsprechende genetische Untersuchungen liessen sich für viele Tiere, Pflanzen und Pilze des Waldes durchführen. Beispielsweise könnten spezielle Insektenarten im Hinblick auf die Vernetzung von Naturwaldreservaten, Altholzinseln und Habitatbäumen oder seltene Gefässpflanzen für eine solche von lichten Wäldern untersucht werden Themen, für die sich kantonale Wald- und Naturschutzämter interessieren, um die Wirksamkeit von Biodiversitätsfördermassnahmen zu überprüfen oder künftige Managementstrategien abzuleiten. Entsprechende Untersuchungen wurden bislang aber nur für wenige Arten, darunter grössere Wildtiere, Vögel und baumbewohnende Moose und Flechten, durchgeführt. Zum Beispiel zeigten Kormann et al (2012), dass beim Auerhuhn (Tetrao urogallus) reger Austausch von Individuen zwischen den kleinen Auerhuhnbeständen im Toggenburg (Kanton St. Gallen) herrscht und dass dabei auch der mehr oder weniger waldfreie und recht stark besiedelte Talboden überwunden wird (Abbildung 2).

\section{Anpassung an den Klimawandel}

Die Reaktion des Waldes auf den Klimawandel ist ein aktuelles Thema: Wie entwickelt sich der Schweizer Wald unter steigenden Temperaturen und zunehmender Trockenheit (Rigling \& Schaffner 2015; Rellstab et al 2016, dieses Heft)? Häufig werden derzeit Modellierungen verwendet, welche aufgrund der heutigen Umweltansprüche von Baumarten zeigen, wie sich deren Verbreitung in der Schweiz unter dem Klimawandel ändern könnte (Pluess et al 2016a). Erörtert wird dabei in erster Linie der mögliche Ausfall der trockenheitsempfindlichen Fichte (Picea abies) als wirtschaftlicher Hauptbaumart im Schweizer Mittelland. Darum wird der Anbau der nicht einheimischen Douglasie (Pseudotsuga menziesii) diskutiert, was aber Befürchtungen von negativen Effekten auf Lebensräume und Biodiversität auslöst (Tschopp et al 2015). Will man deswegen mit einheimischen Baumarten arbeiten, so wird vorgeschlagen, Saatoder Pflanzgut von Beständen aus wärmeren und/ oder trockeneren Regionen der Schweiz, welche vielleicht an höhere Temperaturen oder regional an grössere Trockenheit angepasst sind, in der Nordschweiz auszubringen (Abbildung 3). Durch diese Art von künstlichem Genfluss (sogenannte Translokation oder «Assisted Migration»; Thomas 2011) könnten die Wälder des Mittellandes schneller an den Klimawandel angepasst werden.

Ein Nachteil von Modellierungen der zukünftigen Verbreitung von Baumarten ist, dass sie die Anpassungsfähigkeit bzw. die Ausbreitung und den Genfluss kaum berücksichtigen. Wenn sich Baumarten an höhere Temperaturen und an vermehrte Trockenheit genetisch anpassen können, wie sieht dann ihre künftige Verbreitung aus? Oft wird angemerkt, dass Baumarten eine viel zu lange Generationszeit hätten, als dass sie sich rechtzeitig an den Klimawandel anpassen könnten. Das ist nicht ganz rich- 


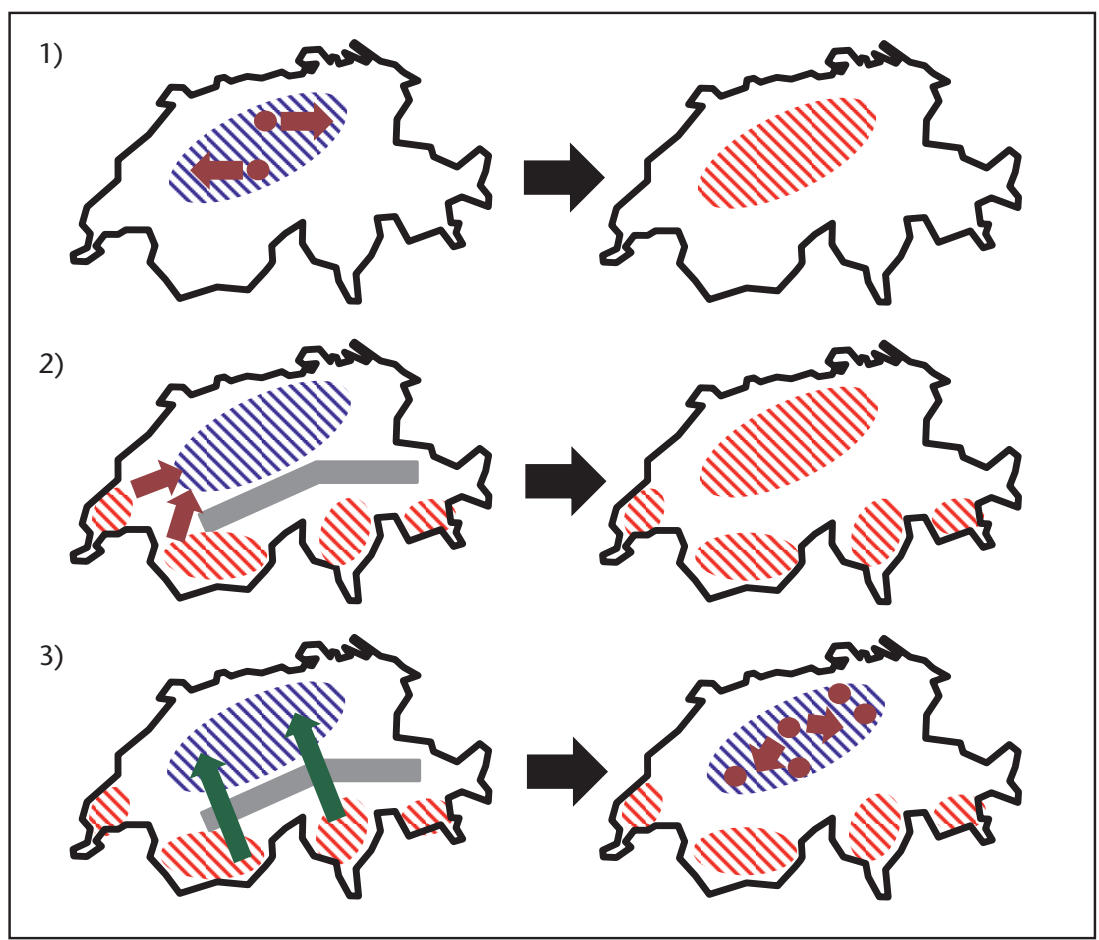

Abb 3 Möglichkeiten der Anpassung von Baumarten an den Klimawandel, dargestellt am Fallbeispiel der heutigen Populationen (blaue Schraffur) im Schweizer Mittelland. 1) Anpassung aufgrund vorhandener genetischer Vielfalt: In den heutigen Beständen findet man einige Bäume, die gut an Trockenheit angepasst sind (rote Punkte). Von diesen aus können sich die entsprechenden Genvarianten durch Samen- und Pollenausbreitung (rote Pfeile) in die anderen Bestände ausbreiten. Diese passen sich durch Gen-Einwanderung ebenfalls an (rote Schraffur). 2) Einwanderung von an Trockenheit angepassten Genvarianten: Bestände aus wärmeren und/oder trockeneren Regionen der Schweiz besitzen bereits an Trockenheit angepasste Genvarianten (rote Schraffur). Durch Samen- und Pollenausbreitung erreichen diese auch das Mittelland. Einige Einwanderungswege werden durch die Alpen blockiert (grauer Balken). 3) Translokation oder «Assisted Migration»: Hier wird an Trockenheit angepasstes Saat- oder Pflanzgut aus wärmeren und/oder trockeneren Regionen der Schweiz im Mittelland ausgebracht (grüne Pfeile). Aus den gepflanzten Beständen (rote Punkte) können sich dann die neuen Genvarianten in andere Bestände ausbreiten. heit selbst, der eigentlichen Genvariante, als auch an der Anpassungsfähigkeit, der Vielfalt dieser Genvarianten, interessiert.

Seit Langem werden zur Untersuchung von Anpassung, etwa bei Fichte, Tanne (Abies alba), Lärche (Larix decidua), Buche oder Eiche, Provenienztests durchgeführt: Keimlinge oder Jungpflanzen verschiedener Herkünfte werden an Orten mit verschiedenen Umweltbedingungen gepflanzt. Wie gut sie mit diesen neuen Umweltbedingungen zurechtkommen, die sich von jenen am Herkunftsort unterscheiden, wird mittels einfach zu messender Merkmale wie etwa des jährlichen Höhenzuwachses erfasst. Statistisch lässt sich dann bestimmen, welcher Anteil der Vielfalt der gemessenen Merkmale genetisch bedingt ist und welcher Anteil durch die Umwelt bestimmt wird. Provenienztests sind langwierig und teuer, da sehr viele Individuen an verschiedenen Orten untersucht werden müssen, damit man statistisch gesicherte Resultate erhält. Man kann mit Provenienztests aber erfassen, welche Herkünfte unter erhöhten Temperaturen bessere Wuchsleistungen erreichen oder bei starker Trockenheit weniger Schäden erleiden. Provenienztests können jedoch die für die Anpassung verantwortlichen Gene selbst nicht bestimmen.

Um die Gene zu bestimmen, die für die Anpassung an gewisse Umweltfaktoren relevant sind, werden molekulargenetische Methoden verwendet. Dabei werden einzelne Gene oder Genvarianten mit verschiedenen Umweltfaktoren statistisch in Verbindung gebracht (Rellstab et al 2016, dieses Heft). Entsprechende genetische Untersuchungen zur Anpassung liessen sich nicht nur bei Bäumen, sondern bei (fast) allen Tieren, Pflanzen und Pilzen des Waldes durchführen.

Braucht es gezielte Pflanzungen, um den Schweizer Wald im Sinne von Assisted Migration an den Klimawandel anzupassen (Abbildung 3)? Nach allem, was wir zurzeit aus Provenienztests und molekulargenetischen Untersuchungen wissen, besitzen Baumarten auch innerhalb von Beständen eine hohe genetische Vielfalt, die für Anpassung, zum Beispiel an sich ändernde Temperaturen, wichtig ist. Die entsprechenden Genvarianten sind im Schweizer Wald verbreitet, wenn auch oft in (noch) geringen Häufigkeiten, da sie unter den heutigen Umweltbedingungen noch nicht vorteilhaft sind (Pluess et al 2016b; Rellstab et al 2016, dieses Heft). Da Pollen und Samen weit wandern, kann man davon ausgehen, dass sich der Schweizer Wald genetisch an den Klimawandel anpassen kann (Abbildung 3) und dass deshalb aus ökologischen Gründen keine speziellen Pflanzungen nötig sind (vielleicht aber aus Gründen der Ökonomie, des Schutzes vor Naturgefahren oder wegen anderer Waldfunktionen; Bradshaw 2004, Pluess et al 2016a).

Bei Pflanzungen muss man zudem bedenken, dass das Pflanzgut meist aus Baumschulen bezogen 
wird, wo es unter günstigen Bedingungen angezogen wird (und dass zumeist nur wenige Herkünfte und eine beschränkte Anzahl Mutterbäume beerntet werden). Gerade die für die erfolgreiche Anpassung in der Natur wichtige Auswahl während des Jugendstadiums fehlt also in Pflanzgärten (St Clair \& Howe 2007). In den Worten der Genetik ausgedrückt: Das Pflanzgut ist einem falschen und geringen Selektionsdruck ausgesetzt. So ist es möglich, dass bei künstlicher Verjüngung mit in Pflanzgärten herkömmlich produziertem, «angepasstem» Pflanzgut die genetische Anpassung an den Klimawandel nicht funktioniert.

Die Schweiz tut einiges für den Schutz der genetischen Vielfalt und der genetischen Ressourcen im Wald: Primat der Naturverjüngung; wenn Pflanzung, dann möglichst mit regionalem Saatgut zur Erhaltung der genetischen Ressourcen; verschiedene Typen von Reservaten etc. (Rigling \& Schaffner 2015). Diese einheimischen genetischen Ressourcen sollten genutzt werden, bevor man an das Pflanzen nicht einheimischer Baumarten denkt.

\section{Biodiversitätsmonitoring}

Molekulargenetische Methoden kommen auch im Rahmen von Biodiversitätsmonitorings zur Anwendung (Schwartz et al 2007), nämlich bei der Identifizierung äusserlich nur schwer bestimmbarer Arten. Hierfür werden einzelne Gensequenzen, also eine kurze Abfolge von Bausteinen des Erbguts, untersucht, die innerhalb einer Art einheitlich, zwischen den Arten aber verschieden sind. Bekannt ist diese Methode als genetisches Barcoding (Holderegger \& Segelbacher 2016). Dafür müssen heutzutage die untersuchten Organismen nicht einmal mehr aufgefunden oder gefangen werden, sondern man kann sogenannte Umwelt-DNA (eDNA; Holderegger $\&$ Segelbacher 2016) verwenden: Kotproben, Federn, Larvenhäute etc. In der Öffentlichkeit bekannt ist der genetische Nachweis von Wolf oder Luchs anhand von Speichelresten an gerissenen Nutztieren.

Die Artbestimmung anhand äusserer Merkmale ist bei Gehölzen in der Regel kein Problem. Doch selbst bei Bäumen kommt genetisches Barcoding zum Einsatz. So lassen sich einheimische Schwarzpappeln (Populus nigra; Abbildung 4) nur durch geschulte Fachleute von nicht einheimischen Hybridpappeln oder von Rückkreuzungen zwischen Schwarz- und Hybridpappeln unterscheiden. Mit genetischem Barcoding ist das aber kein Problem. So lässt sich auch bestimmen, ob sich bei Flussrevitalisierungen auf den neu geschaffenen Offenböden echte Schwarzpappeln oder unerwünschte Hybridpappeln ansiedeln. Glücklicherweise scheint Ersteres in der Schweiz der Fall zu sein (Csencsics \& Holderegger 2016).

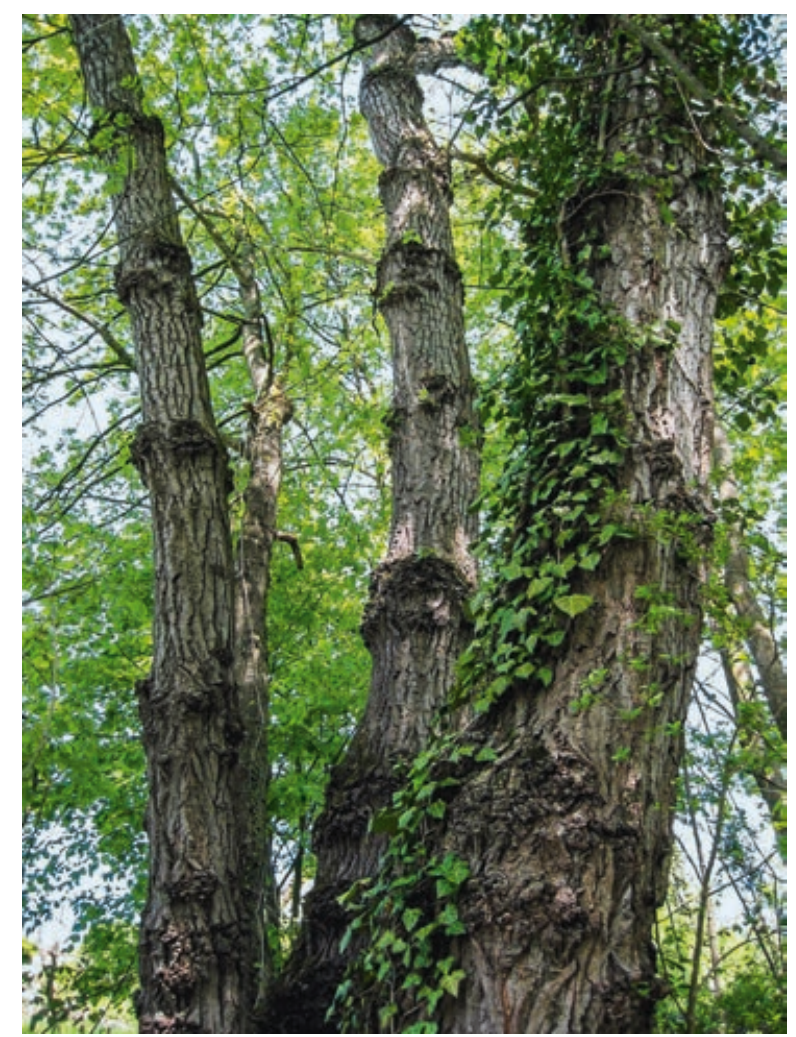

Abb 4 Einheimische Schwarzpappeln (Populus nigra) lassen sich ohne genetische Methoden kaum von nicht einheimischen Hybridpappeln oder Rückkreuzungen mit der Schwarzpappel unterscheiden. Die oft, aber nicht immer vorhandenen Maserknollen sind eines der besten äusserlichen Merkmale zur Erkennung echter Schwarzpappeln. Foto: Daniela Csencsics

Genetisches Barcoding erlaubt auch die Beschreibung jener Biodiversität, die bislang der Erforschung verschlossen war. Ein eindrückliches Beispiel hierfür ist die Untersuchung der Biodiversität im Boden. Dabei wird das gesamte Erbgut aller in einer Bodenprobe vorhandenen Arten mittels Next-Generation Sequencing und Barcoding analysiert. So können Hunderte bis Tausende von Pilzen, Bakterien oder Kleinstlebewesen wie Fadenwürmer (Abbildung 5) in einer Bodenprobe bestimmt werden. Frey \& Hartmann (2013) und Hartmann et al (2014) ha-

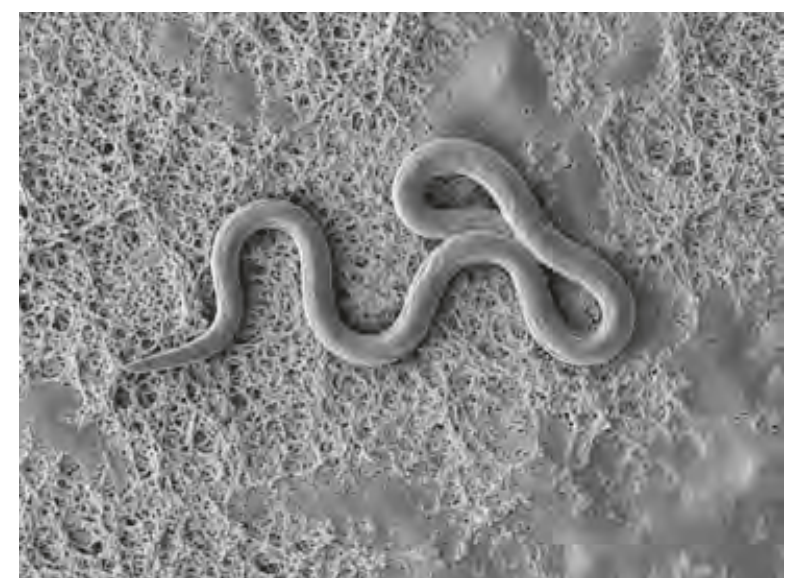

Abb 5 Bodenorganismen wie Fadenwürmer (Nematoden) lassen sich mittels genetischen Barcodings auf Artniveau bestimmen. Foto: Beat Frey 


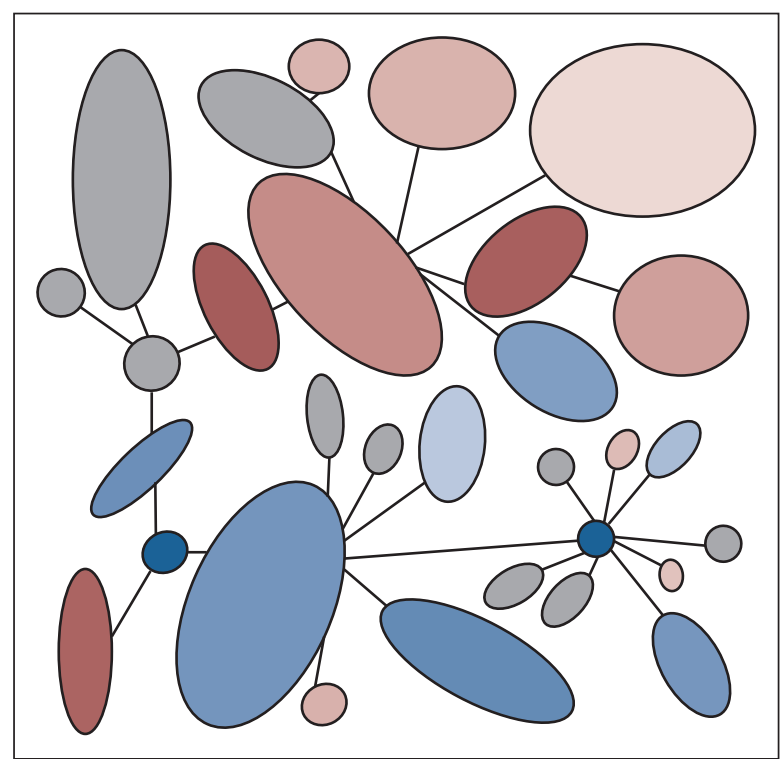

Abb 6 Die Organismenvielfalt im Boden lässt sich mit genetischem Barcoding bestimmen. Das Beispiel zeigt, wie verschiedene Gruppen der Bodenpilze auf Verdichtung des Bodens durch das Befahren mit Holzerntemaschinen reagieren. Die einzelnen Pilzgruppen sind mit farbigen Wolken, ihre verwandtschaftlichen Beziehungen mit Verbindungslinien dargestellt. Blautöne: Bodenpilze, die vor allem in ungestörten Böden häufig sind; Rottöne: Bodenpilze, die vor allem in verdichteten Böden häufig sind; grau: Bodenpilze, die nicht auf Verdichtung reagieren. Bei Bodenverdichtung verschiebt sich die Zusammensetzung der Bodenpilze: blau eingefärbte Pilzgruppen werden seltener, rot eingefärbte häufiger. Vereinfacht nach Hartmann et al (2014).

ben festgestellt, dass Pilze in Waldböden deutlich weniger resistent sind gegenüber Bodenverdichtung durch das Befahren mit Holzerntemaschinen als Bodenbakterien und dass in stark verdichteten Böden noch vier Jahre nach einer Befahrung keine Erholung der Bodenbiodiversität stattgefunden hat. Mykorrhizapilze sind besonders stark betroffen. Änderungen in der Bodenbiodiversität (Abbildung 6) führen auch zu deutlichen Änderungen von Boden- prozessen, zum Beispiel zu erhöhten Methan- und Lachgasemissionen aus verdichteten Böden. Solche Erkenntnisse könnten ohne molekulargenetische Methoden nicht gewonnen werden.

Neben den obigen Beispielen können mit genetischem Barcoding auch Wirkungskontrollen hinsichtlich Biodiversitätsfördermassnahmen durchgeführt, die Vielfalt von Pilzen in Totholzstücken aus Naturwaldreservaten mit solchen aus bewirtschafteten Wäldern verglichen oder die Raumnutzung seltener und scheuer Tierarten wie des Haselhuhns anhand der Losung untersucht werden.

\section{Invasive Schadorganismen}

Globaler Handel und Klimawandel führen dazu, dass invasive Schadorganismen wie Insekten oder Pilze eine immer wichtigere Rolle spielen, mit möglicherweise katastrophalen Folgen für den Wald. Neben altbekannten Krankheiten wie dem Kastanienrindenkrebs (Cryphonectria parasitica) treten jedes Jahr neue Krankheiten und Schädlinge auf - etwa die Kastaniengallwespe (Dryocosmus kuriphilus) oder der Asiatische Laubholzbockkäfer (Anoplophora glabripennis; Abbildung 7, links). Auch hier finden genetische Methoden Verwendung (Hamlin RC 2006, Chown et al 2014). Bei der Bestimmung von Schadorganismen kommt oft genetisches Barcoding zum Einsatz, um die Artbestimmung sicherzustellen. So auch beim Asiatischen Laubholzbockkäfer, dessen Larven sich optisch nur schwer von den Larven anderer (nicht) einheimischer, oft seltener Bockkäfer unterscheiden lassen (Wermelinger et al 2015).

Mit genetischen Methoden können auch die Herkunft und damit die Einführungswege von invasiven Schadorganismen nachvollzogen (Dutech et al 2012) und es kann nach biologischen Bekämpfungsmethoden gesucht werden. Ein aktuelles Bei-
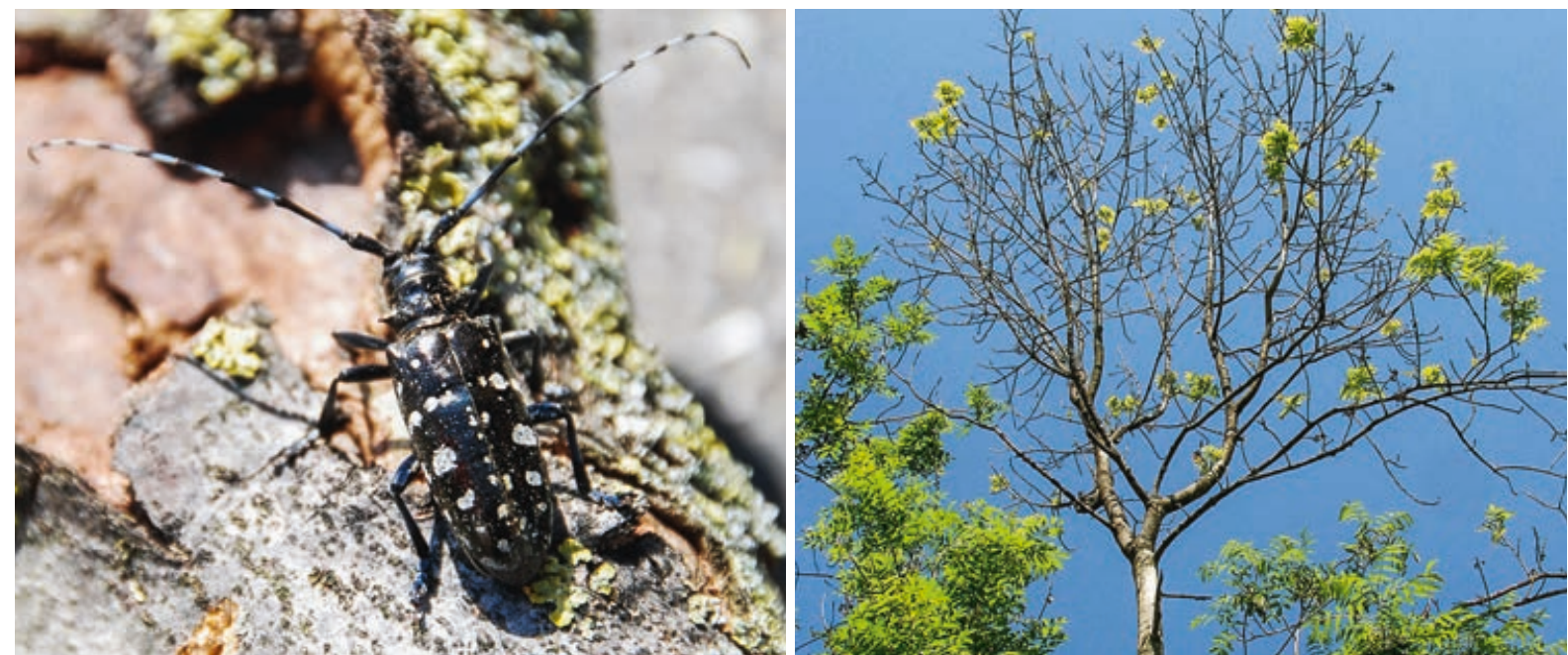

Abb 7 Der Asiatische Laubholzbockkäfer (Anoplophora glabripennis; links) befällt bislang vor allem Stadtbäume in der Schweiz. Seine Larven wurden mit genetischem Barcoding eindeutig identifiziert. Das Eschentriebsterben (rechts), verursacht durch den Pilz Hymenoscyphus fraxineus, mit typischen abgestorbenen Spitzentrieben und verlichteter Krone. Fotos: Waldschutz Schweiz 
spiel hierfür ist das Eschentriebsterben (Abbildung 7, rechts). Seit 2008 hat sich dieses in der Schweiz schnell ausgebreitet und erfasst nun (fast) die ganze Schweiz. Zuerst wurde als Erreger des Eschentriebsterbens die Pilzart Weisses Stengelbecherchen ( $\mathrm{Hy}$ menoscyphus albidus) bestimmt. Doch wie war es möglich, dass eine in der Schweiz schon lange bekannte Pilzart plötzlich grosse Schäden verursacht? Queloz et al (2011) konnten mit molekulargenetischen Methoden zeigen, dass es sich beim Erreger des Eschentriebsterbens tatsächlich um eine neue, fast gleich aussehende Art, $H$. fraxineus, handelt. Vergleiche verschiedener Herkünfte des Erregers mittels genetischer Methoden zeigten zudem, dass $H$. fraxineus aus dem asiatischen Raum nach Europa verschleppt wurde.

Bislang reagiert die Waldwirtschaft mit passiven Methoden auf das Eschentriebsterben. Stark befallene, bereits abgestorbene Bäume werden dort gefällt, wo sie für Verkehrsinfrastruktur oder Waldbesucher eine Gefahr darstellen, und Nothiebe werden durchgeführt, um das Holz frisch befallener Bäume zu ernten, bevor dieses wertlos wird. Dabei kann allerdings die Biodiversität im Wald beeinträchtigt werden: In gewissen Waldgesellschaften ist die Esche charakteristisch und bestandesbildend, und es gibt eine Vielzahl von Moosen und Flechten, die auf die Borke von Eschen angewiesen sind (Rigling et al 2016). Zur Eindämmung des Eschentriebsterbens wird daher auch nach aktiven Methoden gesucht. So könnte man resistentes Pflanzgut züchten, denn es scheint, dass nicht alle Eschen gleichermassen anfällig sind. Im Ausland ist die Forschung zur Züchtung resistenter Eschen bereits im Gange, wobei genetische Methoden naturgemäss eine wichtige Rolle spielen (McKinney et al 2011). In der Schweiz, wo die Waldwirtschaft vor allem auf Naturverjüngung setzt, ist die Züchtung resistenter Eschen die letzte Option. Da die Resistenz gewisser Eschen gegenüber dem Eschentriebsterben mindestens teilweise genetisch bedingt ist, dürfte in befallenen Gebieten - wo ein natürlicher Selektionsdruck herrscht - ein Teil der nachfolgenden Eschengeneration bereits natürlicherweise resistent sein (McKinney et al 2014). So könnte sich der Eschenbestand mittel- bis langfristig wieder von selbst - also ohne spezielle Massnahmen - erholen. In der Schweiz wird auch daran geforscht, ob natürlich vorkommende Viren zur biologischen Bekämpfung des Eschentriebstrebens verwendet werden können (Rigling \& Schoebel 2013) - also ähnlich wie beim Kastanienrindenkrebs, wo seit Jahren ein Virus des Erregerpilzes (Hypovirus von C. parasitica) erfolgreich eingesetzt wird. Auch hier kommen modernste molekulargenetische Methoden zum Einsatz. Dabei werden $H$. fraxineus-Pilze aus aktuell befallenen Eschenbeständen in der Schweiz mit solchen aus Litauen verglichen, wo die Befallswelle des Eschen- triebsterbens bereits vorüber ist. Tatsächlich konnten Viren in $H$. fraxineus festgestellt werden (Schoebel et al 2014). Noch offen ist, ob sich diese für die biologische Bekämpfung eignen.

\section{Ausblick}

Im Rahmen der multifunktionalen Waldwirtschaft sind die Anwendungen genetischer Methoden vielfältig, nicht nur bei Baumarten, sondern auch bei vielen weiteren Organismengruppen. Genetik im Waldbereich hat in der Schweiz aber oft einen anderen Anwendungszweck als im Ausland, da eigentliche Züchtung und (wirtschaftlich relevante) Bereitstellung von Pflanzgut zurzeit keine grosse Rolle spielen. Dies wird sich auch unter dem Klimawandel kaum ändern. Da die Kosten genetischer Methoden sich heute in einem ähnlichen Bereich bewegen wie jene traditioneller Untersuchungen, gilt es, die vielen Anwendungen von Genetik im Wald vermehrt und zielgerichtet zu nutzen, zum Beispiel bei Bäumen zur Suche nach den relevanten Genen zur Anpassung an den Klimawandel, zur Untersuchung der Vernetzung von Natur- und Sonderwaldreservaten ein Prozess, zu dem kaum Wissen vorhanden ist -, zur Bekämpfung von invasiven Arten und ganz allgemein für das Biodiversitätsmonitoring im Wald, betreffe das Bäume, andere Pflanzen, Tiere, Pilze, Flechten oder Schadorganismen. Genetik kann die Türe zu einer mehr funktionalen Erforschung von ökologischen Prozessen im Wald öffnen.

Eingereicht: 6. November 2016, akzeptiert (mit Review): 11. Mai 2016

\section{Literatur}

BACLES CFE, JUMP AS (2011) Taking a tree's perspective on forest fragmentation genetics. Trends Plant Sci 16: 13-18.

BAFU (2012) Strategie Biodiversität Schweiz. Bern: Bundesamt Umwelt. 89 p.

BRADSHAW RHW (2004) Past anthropogenic influence on European forests and some possible genetic consequences. For Ecol Manage 197: 203-212.

CHOWN SL, HODGINS KA, GRIFFIN PC, OAKESHOTT JG, BYRNE M ET AL (2014) Biological invasions, climate change and genomics. Evol Appl 8: 23-46.

CSENCSICS D, HOLDEREGGER R (2016) Origin of natural regeneration of black poplar (Populus nigra L.) and co-occurrence with hybrid poplar offspring. Flora 222: 20-26.

DUTECH C, BARRES B, BRIDIER J, ROBIN C, MILGROOM MG ET AL (2012) The chestnut blight fungus world tour: successive introduction events from diverse origins in an invasive plant fungal pathogen. Mol Ecol 21: 3931-3946.

FREY B, HARTMANN M (2013) Biodiversität von Waldböden - Auswirkungen des Einsatzes von Holzerntemaschinen auf mikrobielle Gemeinschaften. In: Brunner I, Lüscher P, editors. Bodenschutz im Wald: Ziele-Konflikte - Umsetzung: Birmensdorf: Eidgenöss Forsch.anstalt WSL, WSL Ber 6. pp. 61-69.

HAMELIN RC (2006) Molecular epidemiology of forest pathogens: from genes to landscape. Can J Plant Pathol 28: 167-181. 
HARTMANN M, NIKLAUS PA, ZIMMERMANN S, SCHMUTZ S, KREMER J ET AL (2014) Resistance and resilience of the forest soil microbiome to logging-associated compaction. ISME J 8: 226-244.

HOLDEREGGER R, SEGELBACHER G, EDITORS (2016) Naturschutzgenetik. Ein Handbuch für die Praxis. Bern: Haupt. 247 p.

KAMM U, GUGERLI F, ROTACH P, EDWARDS PJ, HOLDEREGGER R (2012) Seltenes und zerstreutes Vorkommen: Auswirkungen auf den Paarungserfolg des Speierlings. Schweiz Z Forstwes 163: 130-136. doi: 10.3188/szf.2012.0130

KORMANN U, GUGERLI F, RAY N, EXCOFFIER L, BOLLMANN K (2012) Parsimony-based pedigree analysis and individualbased landscape genetics suggest topography to restrict dispersal and connectivity in the endangered capercaillie. Biol Conserv 152: 241-252.

KOSKELKA J, BUCK A, TEISSIER DU CROS E, EDITORS (2007) Climate change and forest genetic diversity: implications for sustainable forest management in Europe. Rom: Biodiversity International. $111 \mathrm{p}$.

KRAMER AT, ISON JL, ASHLEY MV, HOWE HF (2008) The paradox of forest fragmentation genetics. Conserv Biol 22: 878-885.

MCKINNEY LV, NIELSEN LR, HANSEN JK, KJAER ED (2011) Presence of natural genetic resistance in Fraxinus excelsior (Oleaceae) to Chalara fraxinea (Ascomycota): an emerging infectious disease. Heredity 106: 788-797.

MCKINNEY LV, NIELSEN LR, COLLINGE DB, THOMSON IM, HAN SEN JK ET AL (2014) The ash dieback crisis: genetic variation in resistance can prove a long-term solution. Plant Pathol 63: 485-499.

PLUESS AR, AUGUSTIN S, BRANG P, EDITORS (2016A) Wald im Klimawandel. Grundlagen für Adaptationsstrategien. Bern: Haupt. 445 p.

PLUESS A, FRANK A, HEIRI C, LALAGÜE H, VENDRAMIN GG ET AL (2016B) Genome-environment association study suggests local adaptation to climate at the regional scale in Fagus sylvatica. New Phytol 210: 589-601.

QUELOZ V, GRÜNIG CR, BERNDT R, KOWALSKI T, SIEBER TN ET AL (2011) Cryptic speciation in Hymenoscyphus albidus. Forest Pathol 41: 133-142.

\section{De nombreuses applications des méthodes génétiques dans la forêt}

La forêt suisse est cultivée proche de la nature et se régénère en grande partie naturellement. Cela présente une bonne base pour la conservation de la diversité génétique des arbres. Pourquoi donc la génétique, quand l'élevage des arbres et la plantation artificielle ne sont guère des buts dans la forêt suisse? En fait, les applications des méthodes génétiques dans la forêt sont plus diverses. Elles sont utilisées pour la détection de réseaux et la surveillance de la performance des activités de réseautage, par exemple, entre les réserves de forêts naturelles, les îlots de sénescence et des arbres-habitats. En utilisant des méthodes génétiques, on peut étudier comment les arbres indigènes et leurs variétés génétiques peuvent être utilisés pour l'adaptation de la forêt au changement climatique. D'autres applications comprennent l'étude de la biodiversité et de l'écologie, par exemple l'influence de la gestion forestière sur la biodiversité du sol, ou la reconnaissance de ravageurs non indigènes et la recherche pour des méthodes de lutte biologique. Dans le cadre de la gestion forestière multifonctionnelle, les méthodes génétiques peuvent donc jouer un rôle important et constituer un emploi utile.
RELLSTAB C, PLUESS AR, GUGERLI F (2016) Lokale Anpassung bei Waldbaumarten: genetische Prozesse und Bedeutung im Klimawandel. Schweiz Z Forstwes 167: 333-340. doi: 10.3188/ szf. 2016.0333

RIGLING A, SCHAFFNER HP, EDITORS (2015) Waldbericht 2015. Bern: Bundesamt Umwelt. 143 p.

RIGLING D, SCHOEBEL C (2013) Neue Perspektiven zur Bekämpfung des Eschentriebsterbens in Europa? Birmensdorf: Eidgenöss Forsch.anstalt WSL. 6 p.

RIGLING D, HILFIKER S, SCHÖBEL C, MEIER F ET AL (2016) Das Eschentriebsterben. Biologie, Krankheitssymptome und Handlungsempfehlungen. Birmensdorf: Eidgenöss Forsch.anstalt WSL, Merkbl Praxis 57. 8 p.

SCHOEBEL C, ZOLLER S, RIGLING D (2014) Detection and genetic characterisation of a novel mycovirus in Hymenoscyphus fraxineus, the causal agent of ash dieback. Infect Genet Evol 28: 78-86.

SCHWARTZ MK, LUIKART G, WAPLES RS (2007) Genetic monitoring as a promising tool for conservation and management. Trends Ecol Evol 22: 25-33.

SEHR EM, FLUCH S (2016) Markerunterstützte Selektion im Forstbereich: theoretisch, praktisch, zukünftig? Schweiz Z Forstwes 167: 341-343. doi: 10.3188/szf.2016.0341

ST CLAIR JB, HOWE GT (2007) Genetic maladaptation of coastal Douglas-fir seedlings to future climates. Glob Chang Biol 13: 1441-1454.

THOMAS CD (2011) Translocation of species, climate change and the end of trying to recreate past ecological communities. Trends Ecol Evol 26: 216-221.

TSCHOPP T, HOLDEREGGER R, BOLLMANN K (2015) Auswirkungen der Douglasie auf die Waldbiodiversität. Schweiz Z Forstwes 166: 9-15. doi: 10.3188/szf.2015.0009

WERMELINGER B, FORSTER B, HÖLLING D, PLÜSS T, RAEMY O ET AL (2015) Invasive Laubholz-Bockkäfer aus Asien. Ökologie und Management. Birmensdorf: Eidgenöss Forsch.anstalt WSL, Merkbl Praxis 50. $16 \mathrm{p}$.

\section{Diverse applications of genetic methods in forest ecosystems}

Swiss forests are managed in a close-to-nature way, and they mainly get naturally rejuvenated. This leads to a forest situation most beneficial for the conservation of the genetic diversity of forest trees. If tree breeding and artificial planting are not main targets in Switzerland, why then using genetics in Swiss forests? In fact, the applications of genetic methods in forests are manifold. They can be used for the identification of connectivity and the evaluation of the success of connectivity measures, e.g., among total reserves, old-growth stands and habitat trees. With genetic methods, one may also study how native tree species and their genetic varieties can best be used for adaptation to climate change. Other applications comprise the investigation of biodiversity and ecology, e.g., the influence of forest management on soil biodiversity, or the diagnosis of invasive pests and pathogens and the search for agents of biological control. In the framework of multifunctional forest management, genetic applications can play an important role, if used in a sensible way. 